\title{
Multiple Scattering of Electromagnetic Waves in Disordered Magnetic Media: Localization Parameter, Energy Transport Velocity and Diffusion Constant
}

\author{
F. A. Pinheiro ${ }^{1}$, A.S. Martinez ${ }^{2}$, and L. C. Sampaio ${ }^{1}$ \\ ${ }^{1}$ Centro Brasileiro de Pesquisas Físicas/CNPq, \\ Rua Dr. Xavier Sigaud, 150, CEP 22290-180, Rio de Janeiro, RJ, Brazil \\ ${ }^{2}$ Faculdade de Filosofia, Ciências e Letras de Ribeirão Preto, Universidade de São Paulo, \\ Av. Bandeirantes 3900, CEP 14040-901, Ribeirão Preto, SP, Brazil
}

Received on 16 August, 2000

\begin{abstract}
We review some of our recent results concerning the single and multiple eletromagnetic scattering by magnetic spherical particles. For a single electromagnetic scattering we show that the magnetic contribution alters, when compared to nonmagnetic scattering, the behavior of the cross sections and mean cosine of the scattering angle $(\langle\cos \theta\rangle)$. For ferromagnetic particles, resonances may occur even in the small-particle limit when the particle radius is much smaller than the wavelength. The resonances increase the cross-sections while $\langle\cos \theta\rangle$ is diminished, and even may become negative. Several quantities such the Ioffe-Regel parameter for localization are calculated for the multiple scattering regime. We show that magnetic scattering favors the observation of localization of electromagnetic waves in three dimensions. Further, this is also verified for dynamical experiments, where we show that the diffusion constant can be very small. Since the magnetic permeability of the scatterers can vary significatively around the Curie-Weiss critical temperature, experiments should be done on the microwave region and the scatterers should be soft ferrites. Some aspects of such experiment are presented.
\end{abstract}

\section{Introduction}

The interest in studying the propagation of electromagnetic (EM) waves in random media has experienced a considerable increase over the last years. After the observation of the coherent backscattering effect,[1] the optical counterpart of electronic weak localization, it has been soon realized that many electronic effects in condensed matter physics have their analog in EM wave systems. Among these, are the photonic Hall effect,[2] the anisotropic light diffusion [3] and the localization of light.[4] Although these phenomena characterize a close analogy between EM waves and electrons, dynamical situations concerning EM and electron propagation reveal fundamental differences between them. [5] In fact, van Albada et al.[6, 7] have shown that, the energy transport velocity $v_{E}$, which characterizes the dynamics of light diffusion through the disordered media, is not equal to the phase velocity $v_{p}$ as in the electronic case. This is because a correction factor in $v_{E}$ (associated with the stored EM energy inside each scatterer due to Mie resonances) should be taken into account in the optical case, in contrast to electronic systems since for electron-impurity scattering this correction is absent.
However, in all multiple scattering phenomena reported so far, including the destruction of the coherent backscattering effect by magneto-optical active materials, $[8,9]$ it is assumed that the scatterers are nonmagnetic. The present paper is thus devoted to investigate some aspects of single and multiple scattering and diffusion of EM waves through magnetic random media. $[10,11,12]$

This paper is organized as follows. In Sec. II we treat both analytically and numerically the EM scattering by a single magnetic scatterer, assumed to be a sphere. It is important to point that several of the main properties of multiple scattering in magnetic media are essentially due to unusual features which characterizes magnetic single scattering, such as preferential backscattering and resonance effects even in the smallparticle limit. In Sec. III these results concerning single scattering will be inserted in the multiple scattering context, where we will investigate the consequences of the introduction of magnetic scatterers in the localization parameter and coherent backscattering effect, in the energy transport velocity and in the diffusion constant. We have shown that the presence of magnetic scatterers induces a global decrease in the localization parameter as well as oscillations in the diffusion con- 
stant. Sec. IV is devoted to the discussion of some experimental aspects and possible applications of our results. Exploring the fact that the magnetic permeability of magnetic scatterers is strongly dependent of an external magnetic field $H_{e x t}$ and typically follows the Curie-Weiss law, we suggest that both the localization parameter and the diffusion constant can be tuned by varying either $H_{\text {ext }}$ or the temperature. Finally, some comments and conclusions are addressed in Sec. V.

\section{Single Scattering}

In order to investigate the influence of magnetic scatterers on the localization parameter $k \ell^{*}$ (where $k$ is the wavenumber and $\ell^{*}$ is the transport mean free path), it is essential to consider the single scattering of EM waves by magnetic particles. In single Mie scattering problem, a plane wave with wavelength $\lambda$ is scattered by a homogeneous magnetic sphere of radius $a$, complex magnetic permeability $\mu_{s}$ and complex electric permittivity $\epsilon_{s}$, leading to a complex refraction index $n_{s}=\left(\epsilon_{s} \mu_{s}\right)^{1 / 2}$. The medium has a real electric permittivity $\epsilon_{m}$ (it does not absorb nor emit light) and vacuum magnetic permeability $\mu_{0}$, which leads to a real refractive index $n_{m}=\left(\epsilon_{m} \mu_{m}\right)^{1 / 2}$. The relative refractive index is $m=n_{s} / n_{m}=(\epsilon \mu)^{1 / 2}$, where $\epsilon=\epsilon^{\prime}+\imath \epsilon^{\prime \prime}$ and $\mu=\mu^{\prime}+\imath \mu^{\prime \prime}$ are the complex relative electric permittivity and the complex relative magnetic permeability, respectively. Both the scatterers and the medium are considered to be nonmagneto-optical active.

Although in standard texts on EM scattering [13, $14,15]$ the relative magnetic permeability $\mu$ is usually assumed to be unitary, this is no longer valid for magnetic particles. In ferromagnetic materials $\mu$ can assume a rather large spectrum of values. For instance, for a nickel bulk at room temperature, $\mu$ is of the order of $10^{6}$ for incident radiation in the microwave range.[16]

The quantities of interest in single scattering are the total cross-section $\sigma$ and anisotropy factor $\langle\cos \theta\rangle$, which can be expressed in terms of the Mie coefficients $a_{n}$ and $b_{n} .[13,14,15]$ In the small-particle limit $(k a \ll 1)$, we have, in the lowest order in $k a$,

$$
\begin{aligned}
& a_{1}=-\frac{\imath(k a)^{3}}{3} \frac{k a \psi_{1}^{\prime}(m k a)-2 \tilde{m} \psi_{1}(m k a)}{k a \psi_{1}^{\prime}(m k a)+\tilde{m} \psi_{1}(m k a)} \\
& b_{1}=-\frac{\imath(k a)^{3}}{3} \frac{\tilde{m} k a \psi_{1}^{\prime}(m k a)-2 \psi_{1}(m k a)}{\tilde{m} k a \psi_{1}^{\prime}(m k a)+\psi_{1}(m k a)},
\end{aligned}
$$

where $\tilde{m}=m / \mu=(\epsilon / \mu)^{1 / 2}$ and $\psi_{n}(z)$ and $\psi_{n}^{\prime}(z)$ are the Ricatti-Bessel function and its derivative with respect to the argument, respectively. It is important to stress the fact that small-particle limit does not mean pointlike scatterers.

To obtain the Rayleigh limit, one has also to ensure that the size of the scatterer is small compared to the wavelength inside the particle $(|m| k a \ll 1)$, which leads to $a_{1}=\gamma(1-\epsilon) /(2+\epsilon)$ and $b_{1}=\gamma(1-\mu) /(2+\mu)$, where $\gamma=-2 \imath(k a)^{3} / 3$.[17] Notice that all the dependence on $\mu$ is in the $b_{1}$ term, which vanishes in the nonmagnetic case. In this case, the leading term in the $b_{1}$ expansion is of the order of $(k a)^{5}$. The anisotropy factor in terms of lowest order in $k a$ is $\langle\cos \theta\rangle=\Re\left(a_{1} b_{1}^{*}\right) /\left(\left|a_{1}\right|^{2}+\left|b_{1}\right|^{2}\right)$. Notice that in magnetic scattering $\langle\cos \theta\rangle$ does not vanish, in contrast to the nonmagnetic case.

It is important to point out that, when $|\mu| \gg 1$, which is the case of ferromagnetic materials, the smallparticle limit must be treated in a different form. In this case, the limit $|m| k a \gg 1$ must be considered using $\psi_{n}(z) \sim \cos [z-(n+1) / 2 \pi]$ in Eqs. (1), leading to:

$$
\begin{aligned}
& a_{1}=-\frac{\imath(k a)^{3}}{3} \frac{p(m, k a)+2 \tilde{m}}{p(m, k a)-\tilde{m}} \\
& b_{1}=-\frac{\imath(k a)^{3}}{3} \frac{\tilde{m} p(m, k a)+2}{\tilde{m} p(m, k a)-1},
\end{aligned}
$$

where $p(m, k a)=k a \tan (m k a)$. Differently from the Rayleigh limit, the $a_{1}$ term also has a magnetic contribution for large values of $|\mu|$. The periodic function $\tan (m k a)$, which depends on $\mu$, is responsible for resonances in $\langle\cos \theta\rangle$. Furthermore, these oscillations are due to the real part of $m$, since $\tan (\imath x)=\imath \tanh (x)$ which is nonperiodic.

To analyze in more detail the problem of single magnetic scattering, we numerically calculate the value of $\langle\cos \theta\rangle$ as a function of the size parameter $k a$ for dielectric $\epsilon^{\prime \prime}=0$ scatterers for three different values of the real part of the relative magnetic permeability $\mu^{\prime}$, as is exhibited in Fig. 1 (for details, see Ref. [10]). In the small-particle limit $(k a \ll 1)$, the scattering is isotropic $(\langle\cos \theta\rangle=0)$ for nonmagnetic scatterers $(\mu=1)$. The appearance of usual Mie resonances in $\langle\cos \theta\rangle$ is observed as $k a$ increases. This situation changes drastically when magnetic scatterers are considered. Notice that EM scattering by magnetic particles is characterized by the nonvanishing value of $\langle\cos \theta\rangle$ even in the small-particle limit, as it can be seen by $\mu^{\prime}=2$ and $\mu^{\prime}=100$ curves. Furthermore, we observe the presence of resonance phenomena for large values of $\mu$ in $\langle\cos \theta\rangle$ even in the small-particle limit, which is absent in the nonmagnetic case. This is in agreement with our analytical result [see Eqs. (2) and comments that follow].

To further investigate EM scattering by magnetic particles in the small-particle limit, in Fig. $2\langle\cos \theta\rangle$ is exhibited as function of both the real $\left(\mu^{\prime}\right)$ and imaginary $\left(\mu^{\prime \prime}\right)$ part of $\mu$ for $k a=0.63$. $\langle\cos \theta\rangle$ has an oscillatory dependence on $\mu^{\prime}$, confirming the presence of resonance phenomena. As $\mu^{\prime}$ increases, the real part of $m$ also increases. A large value of $m$ leads to the building up of standing waves inside the scatterer, which are responsible for the observed resonance phenomena. In addition, notice that $\langle\cos \theta\rangle$ can be negative for some 


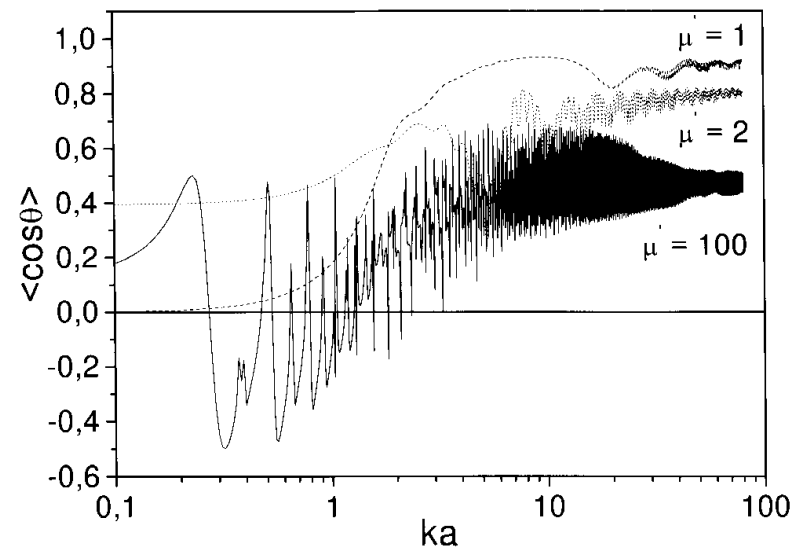

Figure 1. The scattering anisotropy factor $\langle\cos \theta\rangle$, plotted as a function of the size parameter $k a$ for three values of the real part of the relative magnetic permeability: nonmagnetic case $\mu^{\prime}=1$ (dashed curve), $\mu^{\prime}=2$ (dotted curve) and $\mu^{\prime}=100$ (solid curve). The other parameters used are: $\epsilon^{\prime}=1.4161, \epsilon^{\prime \prime}=0$ and $\mu^{\prime \prime}=0$. [reprinted from Pinheiro, Martinez and Sampaio, Phys. Rev. Lett. 84, 1435 (2000), with permission from the American Physical Society].

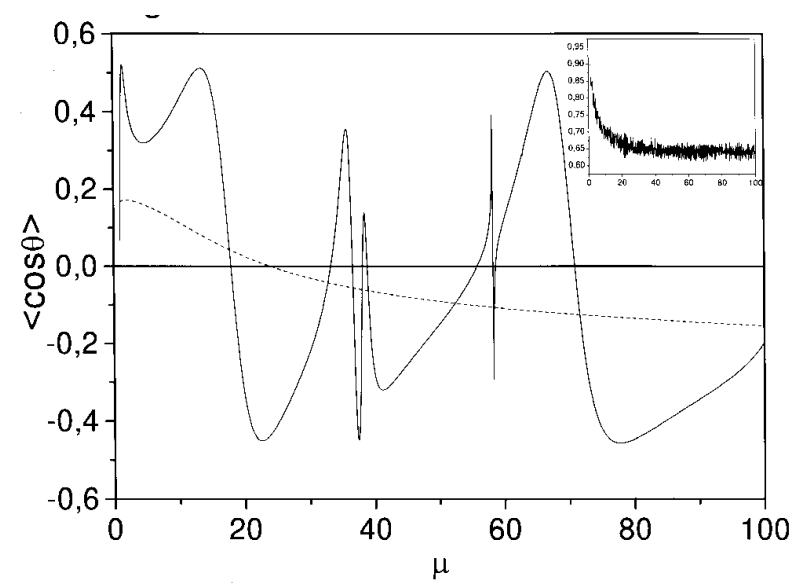

Figure 2. $\langle\cos \theta\rangle$ plotted as a function of both the real $\mu^{\prime}$ (solid curve) and the imaginary $\mu^{\prime \prime}$ (dashed curve) parts of $\mu$ in the small-particle limit $k a=0.63$ and in the largeparticle limit $k a=63$ (inset). The relative dielectric constant is $\epsilon=\epsilon^{\prime}=1.4161$. [reprinted from Pinheiro, Martinez and Sampaio, Phys. Rev. Lett. 84, 1435 (2000), with permission from the American Physical Society].

values of $\mu$, meaning a predominant backward scattering. This preferential backscattering in the smallparticle limit, which is quite unusual in EM scattering by nonmagnetic particles, is due to the significant contribution given by magnetic dipole radiation in magnetic scattering.[17] On the other hand, notice the absence of resonances when $\mu$ has a pure imaginary value: $\langle\cos \theta\rangle$ is a very slowly varying function of $\mu^{\prime \prime}$. We numerically obtain the limiting value $\langle\cos \theta\rangle \sim-0.3$ when $\mu^{\prime \prime} \rightarrow \infty$. This means that a high level of magnetic dissipation of energy contributes only with a constant negative value to $\langle\cos \theta\rangle$ in the small-particle limit. In the inset of Fig. $2\langle\cos \theta\rangle$ is plotted as a function of $\mu^{\prime}$ and $\mu^{\prime \prime}$ for a size parameter $k a=63$, in the socalled large-particle limit. We observe the typical Mie resonances for the real magnetic permeability case, as expected, but the scattering is always preferentially in the forward direction, i.e., $\langle\cos \theta\rangle>0$.

\section{Multiple Scattering}

An important quantity in multiple scattering is the mean length that the wavevector loose memory of the incidence direction. This length is given by the transport mean free path $\ell^{*}=\ell /(1-\langle\cos \theta\rangle)$, where $\ell=$ $1 /\left(\Phi \sigma_{t}\right)$ is the mean free path, $\Phi$ is the number of particle per volume and $\sigma_{t}$ is the total scattering cross section. The localization (Ioffe-Regel) parameter is defined as $k \ell^{*}$ and strong localization is expect when $k \ell^{*} \sim 1$.

\section{III.1 Localization parameter}

In the following, using the above results concerning single magnetic scattering, we numerically calculate $1 /\left(k \ell^{*}\right)$, which is proportional to the width of the backscattering cone, in a magnetic medium for both the small and large particle limits. We only consider the scalar aspect of light propagation. In Fig. 3 we show the dependence of $1 /\left(k \ell^{*}\right)$ on both $\mu^{\prime}$ and $\mu^{\prime \prime}$ in the small-particle limit for dielectric scatterers. Notice the global decrease of the localization parameter induced by the presence of magnetic $(\mu \neq 1)$ scatterers. Furthermore, while $\mu^{\prime \prime}$ gives an almost constant contribution to the cone width, $\mu^{\prime}$ contributes to a fast oscillation. It is also important to point out the wide spectrum of values involved in this oscillation: the cone width can vary over three orders of magnitude when $|\mu|$ varies from 1 to 100 . This interesting pattern of the angular profile of the backscattering cone is essentially due to the fact that the scattering anisotropy can oscillate between positive and negative values as $\mu$ varies.

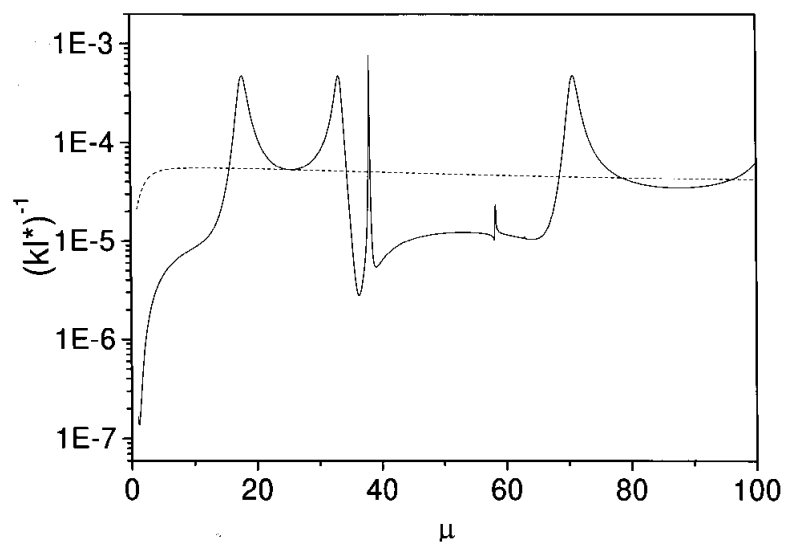

Figure 3. The inverse of the localization parameter $1 /\left(k \ell^{*}\right)$, proportional to the backscattering cone width, plotted as a function of both $\mu^{\prime}$ (solid curve) and $\mu^{\prime \prime}$ (dashed curve) in the small-particle limit $k a=0.63$ for $\epsilon=\epsilon^{\prime}=1.4161$. The wavelength is $\lambda=633 \mathrm{~nm}$ and the concentration of scatterers $\Phi=0.01$. [reprinted from Pinheiro, Martinez and Sampaio, Phys. Rev. Lett. 84, 1435 (2000), with permission from the American Physical Society]. 
The oscillatory behavior for real values of $\mu$ is also present in the large-particle limit (see Fig. 4). Here, not only we observe a global decrease in the localization parameter, when compared with the nonmagnetic case, but also the fact that $k \ell^{*}$ decreases as both $\mu^{\prime}$ and $\mu^{\prime \prime}$ increases until it achieves a saturation minimum value.

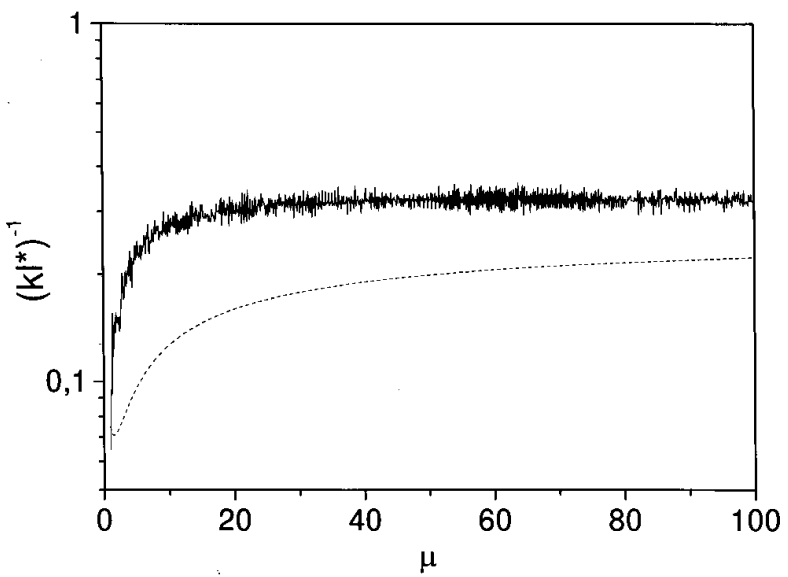

Figure 4. The inverse of the localization parameter $1 /\left(k \ell^{*}\right)$ plotted as a function of both $\mu^{\prime}$ (solid curve) and $\mu^{\prime \prime}$ (dashed curve) in the large-particle limit $k a=63$ for $\epsilon=\epsilon^{\prime}=1.4161$. The wavelength is $\lambda=633 \mathrm{~nm}$ and the concentration of scatterers $\Phi=0.01$. [reprinted from Pinheiro, Martinez and Sampaio, Phys. Rev. Lett. 84, 1435 (2000), with permission from the American Physical Society].

\section{III.2 Energy transport velocity and dif- fusion constant}

The unusual resonant behavior exhibited by EM scattering by magnetic particles in the small-particle limit discussed in Section II has also important implications in the dynamics of the diffusion process. In fact, the diffusion constant $D=v_{E} \ell^{*} / 3$ of EM propagation is strongly affected in the presence of magnetic scatterers, not only through the transport mean free path $\ell^{*}$, as discussed in Section 3.1, but also through the "transport velocity" $v_{E}$. The fact that single scattering by magnetic particles exhibits a characteristic resonant behavior, even in the small-particle limit, will introduce an extra time delay in EM propagation, causing a decrease of $v_{E}$ if compared to the nonmagnetic case. To solve a discrepancy between stationary and dynamic experiments, van Albada et al.,[6, 7] have developed a scalar theory using the low-density approximation, which takes into account a renormalization mechanism of $D$ for classical waves. According to this theory, the transport velocity $v_{E}$ for spherical dielectric Mie scatterers is given by:[7]

$$
\frac{v_{E}}{c_{0}}=\frac{c_{0}}{v_{p}}\left[1+\frac{3 f}{4 x^{2}} \sum_{n=1}^{\infty}(2 n+1) \frac{\mathrm{d}\left(\alpha_{n}+\beta_{n}\right)}{\mathrm{d} x}-\frac{f C}{2}\right]^{-1},
$$

where $c_{0}$ is the velocity of propagation in the vacuum, $f$ is the packing fraction and $v_{p}=c_{0} /(1+f C)^{1 / 2}$ is the phase velocity. The phase angles $\alpha_{n}(k a)$ and $\beta_{n}(k a)$ are defined in terms of the Mie scattering coefficients $a_{n}(k a)$ and $b_{n}(k a)$.[13] The parameter $C(k a)$ is defined according to:

$$
C(k a)=\frac{3}{2(k a)^{3}} \sum_{n=1}^{\infty} \operatorname{Im}\left(a_{n}+b_{n}\right) .
$$

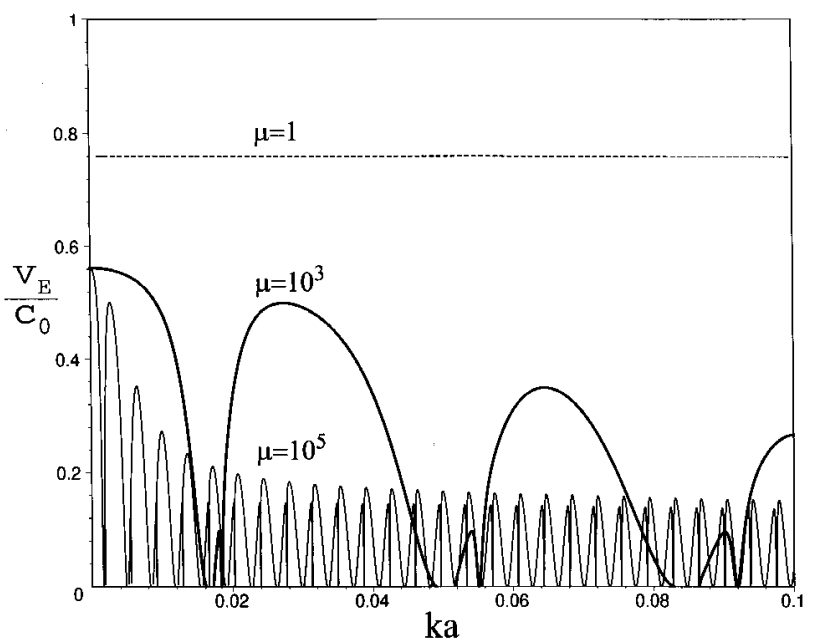

Figure 5. The energy transport velocity $v_{E}$ (normalized by $c_{0}$ ), plotted as a function of $k a$ in the small-particle limit for three values of the (real) magnetic permeability: $\mu=1$ (dashed curve), $\mu=10^{3}$ (bold solid curve) and $\mu=10^{5}$ (solid curve). The packing fraction is $f=0.36$ and $\epsilon=\epsilon^{\prime}=7.29$. [reprinted from Pinheiro, Martinez and Sampaio, Phys. Rev. Lett. ?? , with permission from the American Physical Society].

From Eqs. (2) and (3), we have analytically calculated $v_{E}$ for ferromagnetic Mie scatterers in the smallparticle limit, where resonance phenomena are present in a singular and unusual way. In Fig. $5, v_{E}$ is exhibited as a function of the size parameter $k a$ in the small-particle limit for three different values of $\mu$ for a packing fraction $f=0.36$, the same used in experiments with nonmagnetic scatterers $\mathrm{TiO}_{2}$.[6] For comparison, the curve $\mu=1$ (nonmagnetic case), calculated using the well-known expressions for $a_{1}$ and $b_{1}$ in the Rayleigh limit, $[13,14,15]$ is also shown. Notice that the absence of resonances in the nonmagnetic case $(\mu=1)$ produces an almost constant value of $v_{E}$. This situation changes drastically when magnetic scatterers are taken into account. In this case, $v_{E}$ exhibits sharp drops even in the small-particle limit, in clear contrast to the nonmagnetic case where these drops only occur in the intermediate regime $(k a \sim 1)$ and in the large-particle limit $(k a \gg 1)$.[7] In addition, notice that $v_{E}$ can even vanish for some values of $k a$. As pointed out by van Albada et al.,[6, 7] these sharp drops in $v_{E}$ are essentially due to resonances in the scatterers cross-section, which cause the building up of standing waves inside the scatterers, introducing an extra time delay in EM propagation. 
The influence of the presence of magnetic scatterers on the diffusion constant $D$ in the small-particle $(k a=0.01)$ limit can be seen in Fig. 6 , where $D, v_{E}$ and $\ell^{*}$ are exhibited as a function of the relative magnetic permeability $\mu$. Here one can notice a striking consequence of the introduction of magnetic scatterers in the dynamic properties of EM propagation through disordered media: the presence of oscillations in the diffusion constant $D$. For some values of $\mu$, in particular, one can observe the situation of vanishing diffusion $(D=0)$ of electromagnetic propagation. In addition, it is interesting to point out the damping in the diffusion constant oscillations induced by the increase of the values of $\mu$.

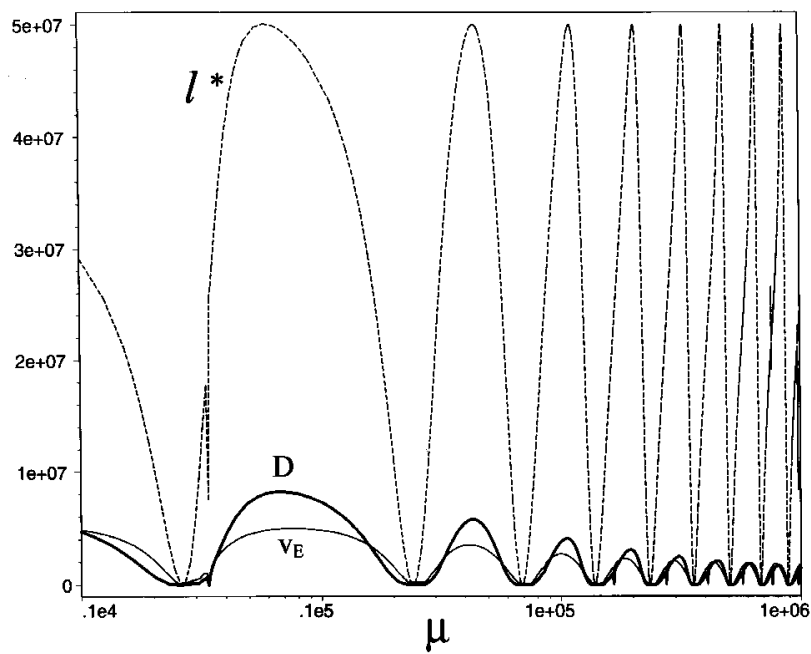

Figure 6 . The transport velocity $v_{E}$ (solid curve), transport mean free path $\ell^{*}$ (dashed curve) and diffusion constant $D$ (bold solid curve), plotted as a function of the (real) (for dielectric ferromagnetic scatterers in the small-particle limit $(k a=0.01)$. In order to exhibit dimensionless quantities $v_{E}, \ell^{*}$ and $D$ are multiplied by $1 / c_{0}, 3 f / 4 a$ and $3 f / 4 a c_{0}$, respectively. For comparison, $v_{E}$ is multiplied by the factor 107. The other parameters used are: $\epsilon^{\prime}=7.29$ and $f=0.36$. [reprinted from Pinheiro, Martinez and Sampaio, Phys. Rev. Lett. ?? , with permission from the American Physical Society]

\section{Experimental Aspects}

Let us now briefly discuss possible situations where our results could be experimentally verified. Most of the soft ferromagnets and ferrimagnets display high magnetic permeability tensor with $\mu$ being strongly dependent on an external applied magnetic field $H_{e x t}$ and on the temperature $T$, i.e., $\mu=\mu\left(H_{e x t}, T\right)$. These facts make all the mentioned properties concerning both single and multiple scattering of EM waves by magnetic particles tunable mechanisms: they can be controlled by the variation of an external parameter, such as $H_{e x t}$ or $T$. For instance, soft ferrites present large values of $\mu$ at microwave frequencies generally below 100 of
$\mathrm{MHz}$ and without loss, which in turn can be easily controlled by the application of an external dc magnetic bias field.[17] For the majority of ferrites, in high frequencies, $\mu$ becomes very small, which turns the utilization of this tunable scattering mechanism fairly difficult at optical frequencies. This kind of restriction to the magnetic permeability have also been employed previously in other contexts involving the interaction between electromagnetic radiation and magnetic materials, such as reported by Sigalas et al., who have recently discussed the possibility of designing photonic crystals with soft magnetic materials.[19]

The magnetic permeability of the vast majority of ferrites typically follows the Curie-Weiss law above the critical temperature, i.e., $\mu \propto\left(T-T_{c}\right)^{-1}$.[17] Thus, a precise tuning of $\mu$ can be performed in this temperature range. As a result, the utilization of magnetic scatterers following the Curie-Weiss susceptibility law offers the possibility to tune the value of both the localization parameter $k \ell^{*}$ and the diffusion constant $D$ by varying the sample temperature. In the large-particle limit, in particular, this fact could be explored in alternative experimental investigations of Anderson localization of EM waves since, as the temperature approaches the Curie-Weiss critical temperature, one can set $k \ell^{*}$ to a minimum value (see Sec. III).

Finally, we suggest that the possibility of controlling the oscillations in the diffusion constant (Sec. III) offers the theoretical basis for the implementation of a magneto-optical device, able to control the transmission of EM waves through disordered magnetic media. Such a device would operate as an optical switch controlled by either the temperature or by an external dc magnetic field (for device applications exploring the transport properties of EM waves through random media see, for instance, Ref. [18]).

\section{Conclusions}

Summarizing, we have studied the influence of magnetic scatterers on single and multiple electromagnetic scattering, and their implications in the coherent backscattering effect, localization parameter, energy transport velocity and diffusion constant. We have shown that, in the small-particle limit, not only single scattering exhibits a forward-backward spatial asymmetry, but also an unusual resonance effect. In multiple scattering context, these unusual aspects concerning single magnetic scattering lead to several interesting consequences, such as the global decrease of the localization parameter, sharp drops in the energy transport velocity even in the small-particle limit and oscillations in the diffusion constant. Furthermore, exploring the fact that the magnetic permeability of magnetic scatterers is strongly dependent on an external magnetic field $H_{e x t}$ and typically follows the Curie-Weiss law, we have suggested 
that both the localization parameter and the diffusion constant can be tuned by varying either $H_{e x t}$ or the temperature.

\section{Acknowledgments}

We thank I. S. Oliveira and L. G. Guimarães for fruitful discussions. This work was supported by the Brazilian agencies CAPES and $\mathrm{CNPq}$.

\section{References}

[1] Y. Kuga and A. Ishimaru, J. Opt. Soc. Am. A 8, 831 (1984); M. P. van Albada and A. Lagendijk, Phys. Rev. Lett. 55, 2692 (1985); P. E. Wolf and G. Maret, ibid. 55, 2696 (1985).

[2] G. L .J. A. Rikken and B. A. van Tiggelen, Nature 381, 54 (1996)

[3] B. A. van Tiggelen, R. Maynard and A. Heiderich Phys. Rev. Lett. 77, 639 (1996); H. Stark and T. C. Lubensky, ibid. 77, 2229 (1996).

[4] D. S. Wiersma, P. Bartolini, A. Lagendijk and R. Righini, Nature 390, 671 (1997).

[5] B. A. van Tiggelen and A. Lagendjik, Phys. Rep. 270, 143 (1996).

[6] M. P. van Albada, B. A. van Tiggelen, A. Lagendijk and A. Tip, Phys. Rev. Lett. 66, 3132 (1991);

[7] B. A. van Tiggelen, A. Lagendijk, M. P. van Albada, and A. Tip, Phys. Rev. B 45, 12233 (1992).

[8] R. Lenke and G. Maret, Phys. Scripta 49, 605 (1993).
[9] A. S. Martinez and R. Maynard, Phys. Rev. B 50, 3714 (1994).

[10] F. A. Pinheiro, A. S. Martinez and L. C. Sampaio, Phys. Rev. Lett. 84, 1435 (2000).

[11] F. A. Pinheiro, A. S. Martinez and L. C. Sampaio, Electromagnetic Scattering by Small Magnetic Particles (to appear in: Journal of Magnetism and Magnetic Materials).

[12] F. A. Pinheiro, A. S. Martinez and L. C. Sampaio, Vanishing of Energy Transport Velocity and Diffusion Constant of Electromagnetic Waves in Disordered Magnetic Media (to appear in: Phys. Rev. Lett.).

[13] H. C. van de Hulst, Light Scattering by Small Particles (Dover, New York, 1980).

[14] M. Kerker, The Scattering of Light and Other Electromagnetic Radiation (Academic Press, New York, 1969).

[15] C. F. Bohren and D. R. Huffman, Absorption and Scattering of Light by Small Particles (John Wiley, New York, 1983).

[16] C. W. Chen, Magnetism and Metallurgy of Soft Magnetic Materials (Dover, New York, 1986).

[17] M. Kerker, D. S. Wang and C. L. Giles, J. Opt. Soc. Am. 73, 765 (1983).

[18] A. Z. Genack and N. Garcia, J. Opt. Soc. Am. B 10, 408 (1993).

[19] M. M. Sigalas, C. M. Soukoulis, R. Biswas, and K. M. Ho, Phys. Rev. B 56, 959 (1997). 